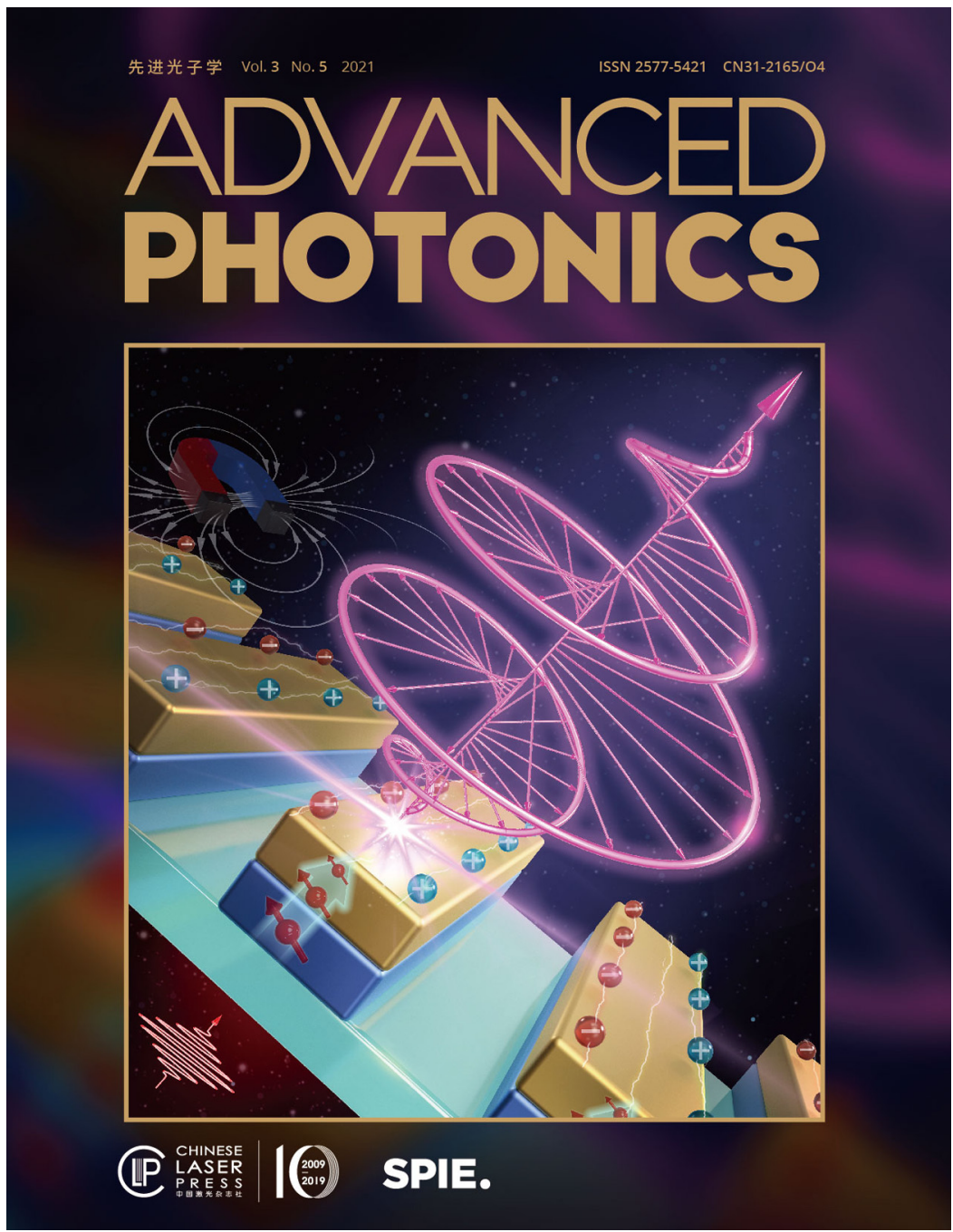

\title{
About the cover: Advanced Photonics Volume 3, Issue 5
}

The image on the cover for Advanced Photonics Volume 3 Issue 5 depicts a novel spintronic-metasurface terahertz emitter, developed by researchers from Fudan University. Composed of alternating magnetic heterostructures, the emitter allows efficient, flexible generation and manipulation of chiral terahertz waves. Terahertz radiation is generated by exciting the emitter with laser pulses under an oriented external magnetic field. Transverse anisotropic confinement of the laser-induced charge currents imposed by the metasurface structure leads to chiral terahertz-wave emission.

The image is based on original research presented in the report by Changqin Liu, Shunjia Wang, Sheng Zhang, and Qingnan Cai, et al., "Active spintronic-metasurface terahertz emitters with tunable chirality," Adv. Photonics 3(5), 056002 (2021), doi: 10.1117/1.AP.3.5.056002. 